\title{
31. Length Regulation of Thin Filaments without Nebulin
}

\author{
By Kenji YASudA, ${ }^{*)}$ Hideaki FujITA, ${ }^{* *)}$ Yasutake FuJIKI, ${ }^{* *)}$ \\ and Shin'ichi ISHIWATA**)
}

(Communicated by Setsuro EBASHI, M. J. A., Nov. 14, 1994)

\begin{abstract}
Recent work has suggested that the length of thin filaments in skeletal muscles is determined by a protein ruler, nebulin, located along the long axis of thin filaments. To examine the function of nebulin, the length distribution of the thin filaments was investigated by staining actin filaments with fluorescent rhodamine-phalloidin in rabbit cardiac muscles, which do not contain nebulin, and in skeletal muscles, which contain nebulin, by laser scanning confocal microscopy. The microscopic observation showed a difference in staining patterns between cardiac and skeletal muscle fibers when the staining was done without chemical fixation. This suggests that nebulin suppresses the attachment of phalloidin to actin filaments. Analysis of fluorescence distribution showed that the length deviation of thin filaments in the cardiac muscle was as small as that in the skeletal muscle. This indicates that the length of the thin filaments is regulated even without nebulin.
\end{abstract}

Key words: Cardiac muscle; skeletal muscle; thin filaments; rhodamine-phalloidin; nebulin; laser scanning confocal microscopy.

Introduction. Earlier studies on muscle structures using the electron microscope showed that both thin and thick filaments are of uniform lengths. ${ }^{1), 2}$ Since then, there have appeared several reports showing that the thin filaments have broad length distribution in rat and frog cardiac muscles ${ }^{3), 4)}$ and even in rat skeletal muscles. ${ }^{5}$

Recently, nebulin, a large protein being specifically present in vertebrate skeletal muscles, has been suggested to be a length regulator for thin filaments. ${ }^{6-11)}$ Wang's group reported a correlation between the molecular weight of nebulin and the thin filament length in several skeletal muscle tissues extracted from chickens, rabbits, humans and snakes in which the filament length was determined within the standard deviations of 0.05 $\mu \mathrm{m} .{ }^{7)}$

However, nebulin is absent in the cardiac muscle; ${ }^{6), 12), 13)}$ although nebulin-sized proteins exist, the amount present in the muscle is small and there is no cross-reactivity with anti-nebulin antibodies. ${ }^{6)}$

In this study, the length distribution of thin filaments is examined by observing actin filaments stained with fluorescent rhodamine-phalloidin $(\mathrm{RhPh})^{14)}$ under a confocal microscope to clarify the role of nebulin as a length determining ruler. The rabbit cardiac papillary and skeletal psoas muscle were chosen as examples suitable for comparison.

Materials and methods. Solutions. Solution A, $0.15 \mathrm{M} \mathrm{KCl}, 1 \mathrm{mM} \mathrm{MgCl}_{2}, 10 \mathrm{mM}$ 3-(N-morpholino) propanesulfonic acid (MOPS) (pH 7.0), $1 \mathrm{mM}$ EGTA, $10 \mathrm{mM}$ DTT, and 1 $\mathrm{mM}$ leupeptin; solution $\mathrm{B}$, solution A containing 1\% Triton-X100; solution C, solution A containing $33 \mu \mathrm{M} \mathrm{RhPh}$; solution $\mathrm{D}$, solution A containing $3 \%$ formaldehyde; solution $\mathrm{E}, 0.1$ $\mathrm{M} \mathrm{KCl}, 2 \mathrm{mM} \mathrm{MgCl} 2,2 \mathrm{mM}$ MOPS (pH 7.0), $1.5 \mathrm{mM} \mathrm{NaN}_{3}$, and $3.3 \mu \mathrm{M} \mathrm{RhPh}$; solution F, $25 \mathrm{mM} \mathrm{KCl}, 4 \mathrm{mM} \mathrm{MgCl} 2,25 \mathrm{mM}$ imidazole- $\mathrm{HCl}$ (pH 7.4), $1 \mathrm{mM}$ EGTA, and $30 \mathrm{mM}$ DTT.

*) Advanced Research Lab., HITACHI Ltd., Hatoyama, Saitama 350-03, Japan.

**) Dept. of Physics, School of Science and Engineering, Waseda Univ., Shinjuku-ku, Tokyo 169, Japan. 
$\mathrm{RhPh}$ was purchased from Molecular Probe Inc. (Eugene OR, U.S.A.); catalase and glucose oxydase were from SIGMA Chemical Co. (St. Louis, Mo., U.S.A.); EGTA and MOPS were from Dojindo Laboratories (Kumamoto); DTT was from Wako Pure Chemical Industries, Ltd. (Osaka). Other chemicals were of reagent grade.

Cardiac and skeletal muscle fibers stained with rhodamine-phalloidin. Two types of preparation were examined for $\mathrm{RhPh}$ staining, i.e., staining without formaldehyde fixation (NF preparation) or staining after formaldehyde fixation ( $\mathrm{FF}$ preparation) of rabbit cardiac papillary and skeletal psoas muscles glycerinated in $50 \%(\mathrm{v} / \mathrm{v})$ glycerol containing $0.5 \mathrm{mM} \mathrm{NaHCO}{ }_{3}, 5 \mathrm{mM}$ EGTA, and $1 \mathrm{mM}$ leupeptin for more than 3 weeks at $-20^{\circ} \mathrm{C}$. First, a single skeletal or a small bundle of cardiac glycerinated fibers were mounted on a glass slide; glycerol was washed out with solution A and the fibers were immersed in solution $\mathrm{B}$ for $30 \mathrm{~min}$ at $4^{\circ} \mathrm{C}$ to remove the membrane system. For NF preparation, solution $\mathrm{B}$ was substituted for solution $\mathrm{C}$, and the fibers were stained with $\mathrm{RhPh}$ overnight at $4^{\circ} \mathrm{C}$ and then free $\mathrm{RhPh}$ was washed out with solution $\mathrm{A}$ so that the fibers could be microscopically observed. For FF preparation, solution B was substituted for solution D instead of solution $\mathrm{C}$ and the fibers were immersed for $30 \mathrm{~min}$ at $4^{\circ} \mathrm{C}$. After formaldehyde fixation, the fibers were stained with $\mathrm{RhPh}$ in solution $\mathrm{C}$ overnight at $4^{\circ} \mathrm{C}$ and then free $\mathrm{RhPh}$ was washed out with solution A to allow microscopic observation.

Single actin filaments stained with rhodamine-phalloidin. Actin was prepared from acetone powder which had been obtained from a rabbit leg and a back white muscle according to a standard procedure. ${ }^{15)}$ For $\mathrm{RhPh}$ staining, $0.1 \mathrm{mg} / \mathrm{ml}$ G-actin was polymerized in solution $\mathrm{E}$ overnight at $4^{\circ} \mathrm{C}$ in the dark. Just before microscopic observation, $30 \mu \mathrm{g} / \mathrm{ml}$ heavy meromyosin in solution $\mathrm{F}$ was infused into the narrow space between a silanizing glass slide and a cover slip, and then $0.5 \mathrm{mg} / \mathrm{ml}$ bovine serum albumin in solution $\mathrm{F}$ was infused. Then $\mathrm{F}$-actin (final concentration, $0.1 \mu \mathrm{g} / \mathrm{ml}$ ) diluted in solution $\mathrm{F}$ containing $0.04 \mathrm{mg} / \mathrm{ml}$ catalase, $0.22 \mathrm{mg} / \mathrm{ml}$ glucose oxydase, and $4.5 \mathrm{mg} / \mathrm{ml}$ glucose was infused for microscopic observation. ${ }^{16)}$

Laser scanning confocal microscopy. Both muscle fibers and actin filaments stained with $\mathrm{RhPh}$ were observed using a laser scanning confocal microscope system (LSMGB200, OLYMPUS Co., Tokyo). The light source was a $25 \mathrm{~mW}$ Ar laser of $488 \mathrm{~nm}$. A $550 \mathrm{~nm}$ long-pass filter was used to detect the fluorescence of RhPh. A PLAPO 60X WLSM [1.0 NA] objective lens was used. Scanning time to take one picture was $16 \mathrm{~s}$. The fluorescence intensity was recorded in a $1024 \times 768$ frame memory with an intensity range of 256 steps. The intensity profile was obtained by scanning along a line with the width of 10 pixels on the $\mathrm{X}-\mathrm{Y}$ plane; the depth of the $\mathrm{X}-\mathrm{Y}$ plane was changed along the $\mathrm{Z}$ axis by moving the sample stage using a piezo actuator.

Data analysis of thin filament fluorescent images. The fluorescence intensity profiles of the thin filaments obtained from confocal images of the I-Z-I brush, a bundle of thin filaments, were compared with a model calculated using a convolution method. The intensity $\mathrm{I}(\mathrm{x})$ of the thin filaments at position $\mathrm{x}$ from the $\mathrm{Z}$ line was calculated using $\mathrm{I}(\mathrm{x})$ $=\mathrm{F}^{-1}(\mathrm{~F}(\mathrm{~T}(\mathrm{x})) \cdot \mathrm{F}(\mathrm{A}(\mathrm{x})))$, where $\mathrm{F}$ and $\mathrm{F}^{-1}$ are, respectively, the Fourier and Fourier inverse transformation functions; $T(x)$ shows a relative proportion of existence of thin filaments $\left(\mathrm{T}\left(\mathrm{x}<\mathrm{L}_{0}\right)=1\right.$ and $\mathrm{T}\left(\mathrm{x}>\mathrm{L}_{0}\right)=0$ when the length of the thin filaments is uniformly $\left.\mathrm{L}_{0}\right) ; \mathrm{A}(\mathrm{x})$ is a relative intensity profile of a point light source (in the present work $\mathrm{RhPh}$-stained single actin filaments were selected as a point light source).

Results and discussion. Confocal images of cardiac and skeletal muscle fibers. First, the stained regions of $\mathrm{NF}$ and $\mathrm{FF}$ prepared cardiac and skeletal muscle fibers were compared with the use of a confocal microscope. In the cardiac muscle, the entire thin filament region was stained in both NF (Figs. 1a, 2a) and FF (Figs. 1b, 2b) preparations. In the skeletal muscle, only the ends of the thin filaments, i.e., the pointed end at the 

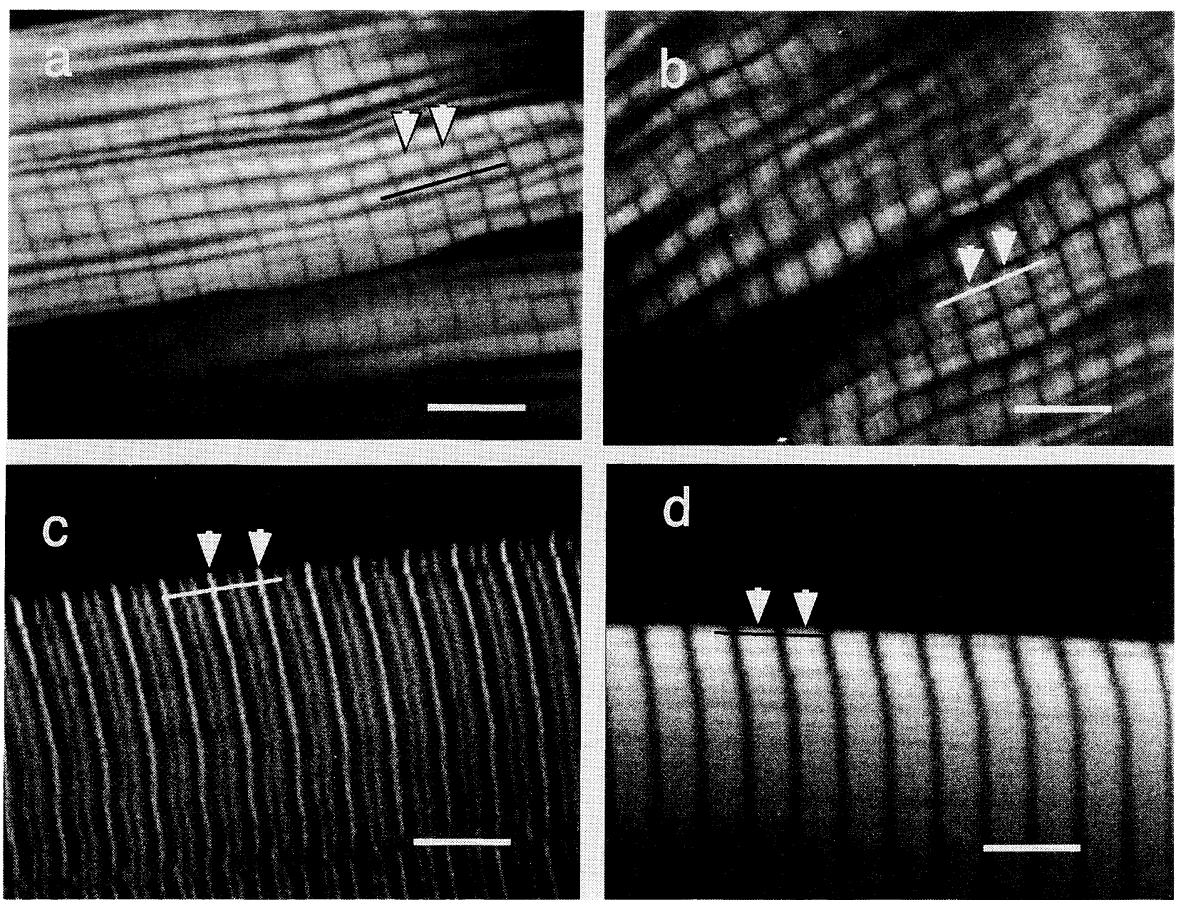

Fig. 1. Confocal fluorescence images of cardiac and skeletal muscle fibers. Actin filaments were made visible by rhodamine-phalloidin staining without (a and c) or after $(b$ and $d$ ) formaldehyde fixation. Micrographs $a$ and $b$ are of the cardiac muscle and $c$ and $\mathrm{d}$ are of the skeletal muscle. The fluorescence intensity differs depending on the gain of the photomultiplier. The intensity profile was obtained along a thin line of each fiber (cf. Fig. 2). Arrows indicate the position of the $\mathrm{Z}$ lines, and the scale bars are $5 \mu \mathrm{m}$.

center of each sarcomere and the barbed end at the $\mathrm{Z}$ line, were stained in the NF preparation (Figs. 1c, 2c), although the entire thin filament region was stained in the FF preparation (Figs. 1d, 2d). Szczesna and Lehrer ${ }^{17)}$ also reported that the staining pattern in skeletal myofibrils, which was initially an NF type, changed to an FF type after staining for $2-3 \mathrm{~h}$ in a rigor solution at low $\mathrm{pH}$. These results suggest that nebulin inhibits $\mathrm{RhPh}$ staining on actin filaments by binding along the long axis. The accessibility of RhPh to its binding site on thin filaments, which is suppressed by nebulin, seems to be modified by the chemical fixation or solvent conditions.

Confocal images of actin filaments. The fluorescence intensity profiles were obtained for single actin filaments regarded as a point light source. Actin filaments were fixed on a glass surface through rigor bonds with heavy meromyosin molecules adsorbed on the glass surface. The intensity profiles were obtained along the $\mathrm{X}$ and $\mathrm{Z}$ axes (Figs. 3a, b). A half width of the former profile was $0.29 \mu \mathrm{m}$ and that of the latter was $2.57 \mu \mathrm{m}$; these values indicate a spatial resolution of the microscopic system. The spatial resolution along the $\mathrm{Z}$ axis was sufficiently narrow to obtain the intensity profiles of the I-Z-I brush in muscle fibers.

Length distribution of thin filaments in muscle fibers. Fluorescence intensity profiles of FF prepared cardiac and skeletal muscles were analyzed using the convolution method described above. Data could be simulated by assuming a uniform length of 1.05 to $1.1 \mu \mathrm{m}$ in the cardiac muscle (Fig. 5a), and 1.0 to $1.1 \mu \mathrm{m}$ in the skeletal muscle (Fig. 5b). The latter 

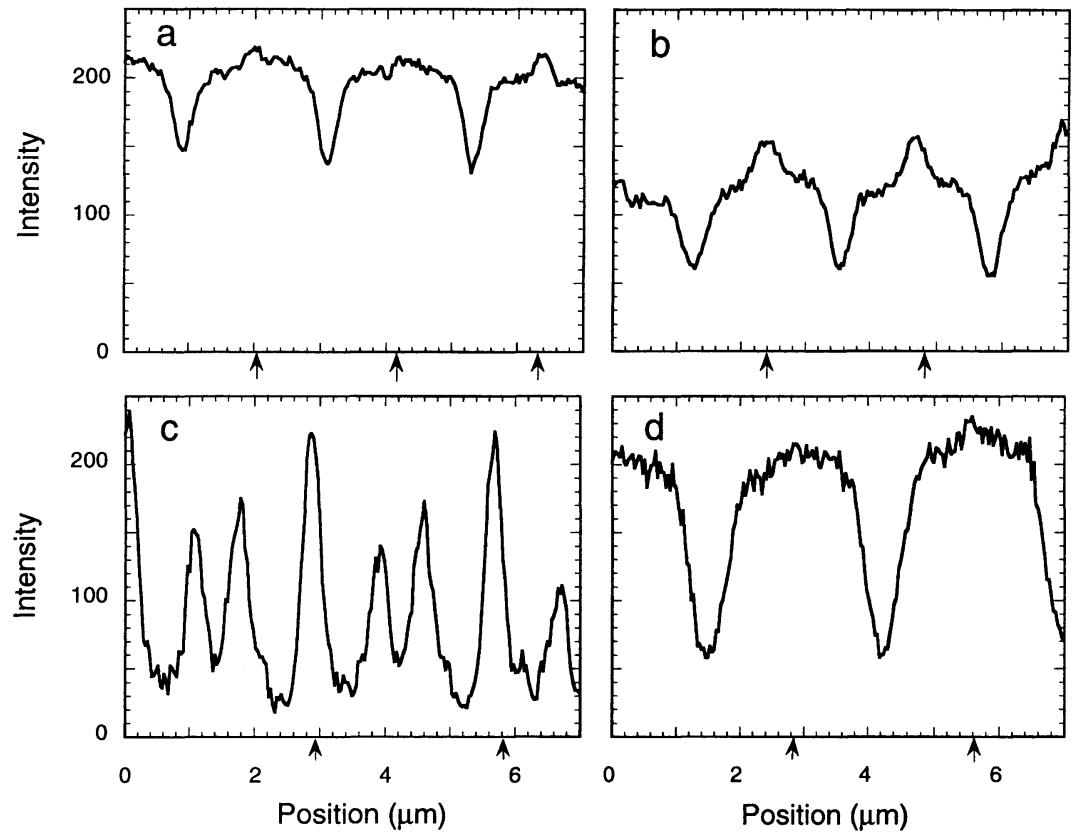

Fig. 2. Fluorescence intensity profiles of cardiac and skeletal muscle fibers stained with rhodamine-phalloidin. Intensity profiles in a to $d$ were obtained along the thin lines indicated in Figs. 1a to d, respectively. Arrows indicate the position of the $\mathrm{Z}$ lines. The ordinate is the fluorescence intensity in arbitrary units (a. u.) and the abscissa represents the position along the long axis of the myofibrils.
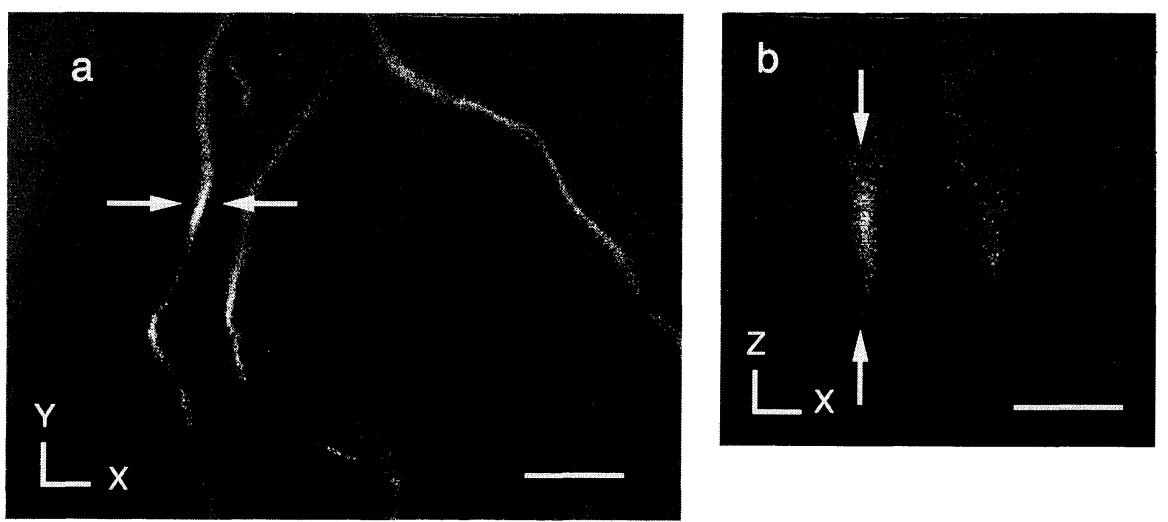

Fig. 3. Confocal fluorescence images of single actin filaments stained with rhodaminephalloidin. Image $a$ is on the $\mathrm{X}-\mathrm{Y}$ plane; image $\mathrm{b}$ is on the $\mathrm{X}-\mathrm{Z}$ plane constructed using image processing from a series of images on the $\mathrm{X}-\mathrm{Y}$ plane obtained along the $\mathrm{Z}$ axis. Arrows, see Fig. 4. Scale bars are $5 \mu \mathrm{m}$ in a and $2 \mu \mathrm{m}$ in b.

deviation was within the same range as that reported previously. ${ }^{7)}$ Concerning the length distribution in the cardiac muscle, a previous electron microscopic observation showed a broad distribution ranging from 0.6 to $1.1 \mu \mathrm{m}$ in rat atrial trabeculae and a somewhat narrower distribution ranging from 0.9 to longer than $1.1 \mu \mathrm{m}$ in rat papillary muscles. ${ }^{3), 4)}$ Our results not only support the latter observation but, moreover, indicate that the width 

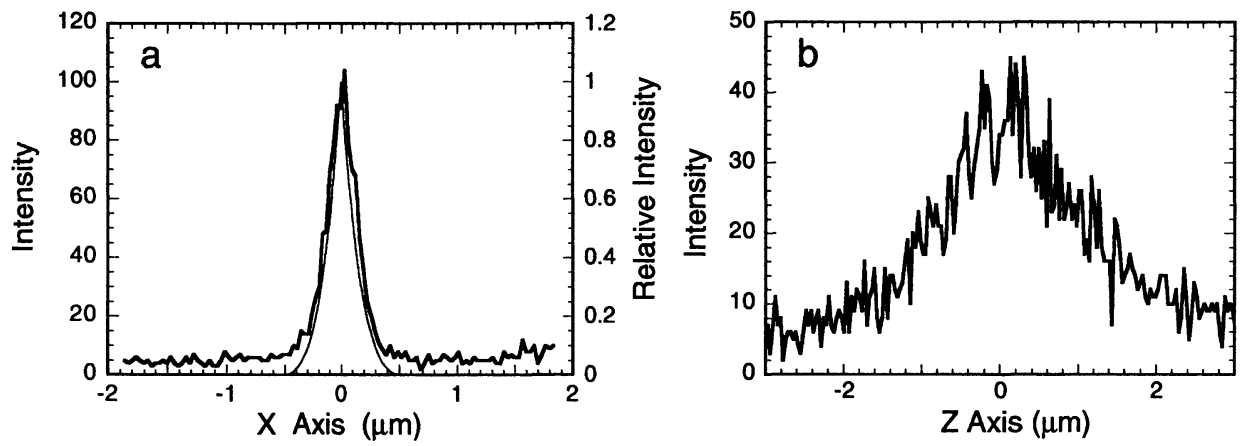

Fig. 4. Fluorescence intensity profiles of single actin filaments stained with rhodaminephalloidin. Intensity profiles were obtained by measuring intensity (a. u.) between the two arrows indicated in Fig. 3. Graph a is the intensity profile along the $\mathrm{X}$ axis on the $\mathrm{X}-\mathrm{Y}$ plane shown in Fig. 3a; the thin line was obtained by smoothing the data profile, which is used for convolution analysis in Fig. 5. Graph $\mathrm{b}$ is the intensity profile along the $\mathrm{Z}$ axis on the $\mathrm{X}-\mathrm{Z}$ plane shown in Fig. 3b.
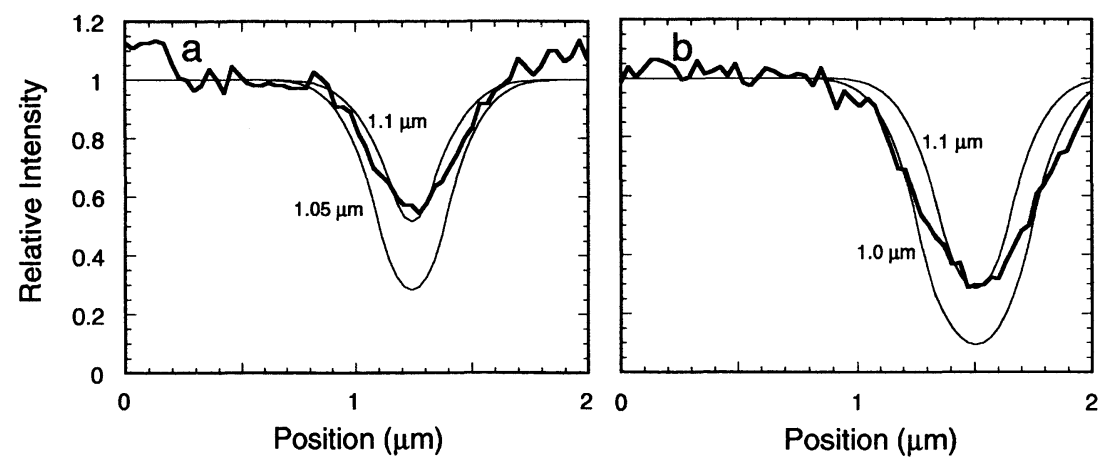

Fig. 5. Analysis of the thin filament length distribution in cardiac (a) and skeletal (b) muscles. Thick lines in $a$ and $b$ indicate the relative intensity profiles obtained from Figs. $2 \mathrm{~b}$ and $\mathrm{d}$, respectively. The thin lines indicate the intensity profiles calculated from the convolution method using the intensity profile of an actin filament shown by the thin line in Fig. 4a and assuming that the thin filaments are of uniform lengths of 1.0, 1.05, and 1.1 $\mu \mathrm{m}$ as shown in the figures.

of the distribution in the papillary muscle is similar to that in the skeletal muscle.

Nebulin is not a length regulator of thin filaments. The present work demonstrated that the length of the thin filaments in rabbit cardiac papillary muscles, which do not contain nebulin, is determined within the spatial resolution of the microscope as in the case of the skeletal psoas muscle, which contains nebulin. This result indicates that nebulin is not a main length regulator of the thin filaments, although we do not deny the possibility that nebulin plays an essential role for length determination within molecular precision in collaboration with a capping protein. ${ }^{15)}$ Finally, we should mention that it is desirable to examine whether the length distribution of the thin filaments in rat skeletal muscle is so broad as reported previously ${ }^{5)}$ by using the technique described here.

Acknowledgments. We thank Dr. Hideki Kanbara of Hitachi, Ltd. for giving us the opportunity to use a confocal microscope system, Dr. Kazuo Takeda of Hitachi, Ltd. for his 
valuable suggestion of using the convolution method for estimating thin filament length, and Dr. Shin-ichiro Umemura of Hitachi, Ltd. for his continuous encouragement. This study was supported in part by Grants-in-Aids for Science Research and for Scientific Research on Priority Areas from the Ministry of Education, Science, and Culture of Japan.

\section{References}

1) Huxley, H. E.: J. Mol. Biol., 7, 281-308 (1963).

2) Page, S. G., and Huxley, H. E.: J. Cell Biol., 19, 369-390 (1963).

3) Robinson, T. F., and Winegrad, S.: Nature (Lond.), 267, 74-75 (1977).

4) - : J. Physiol., 286, 607-619 (1979).

5) Traeger, L., and Goldstein, M. A.: J. Cell Biol., 96, 100-103 (1983).

6) Wang, K., and Wright, J.: ibid., 107, 2199-2212 (1988).

7) Kruger, M., Wright, J., and Wang, K.: ibid., 115, 97-107 (1991).

8) Labeit, S. et al.: FEBS Lett., 282, 313-316 (1991).

9) Trinick, J.: ibid., 307, 44-48 (1992).

10) Wright, J., Huang, Q.-Q., and Wang, K.: J. Muscle Res. Cell Motil., 14, 476-483 (1993).

11) Pfuhl, M., Winder, J., and Pastore, A.: EMBO J., 13, 1782-1789 (1994).

12) Locker, R. H., and Wild, D. J. C.: J. Biochem., 99, 1473-1484 (1986).

13) Hu, D. H., Kimura, S., and Maruyama, K.: ibid., 99, 1485-1492 (1986).

14) Faulstich, H. et al.: J. Muscle Res. Cell Motil., 9, 370-383 (1988).

15) Ishiwata, S., and Funatsu, T.: J. Cell Biol., 100, 282-291 (1985).

16) Kron, S. J. et al.: Methods in Enzymology, 196, 399-416 (1991).

17) Szczesna, D., and Lehrer, S. S.: J. Muscle Res. Cell Motil., 14, 594-597 (1993). 